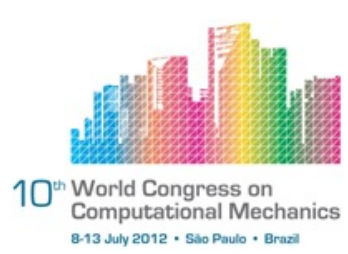

\title{
DEVELOPMENTS AND APPLICATIONS OF THE TECHNIQUE OF THE COMPOSITE MESH
}

\author{
M. B. Bergallo ${ }^{1}$, C. A. Neuman ${ }^{1}$, V. E. Sonzogni ${ }^{2}$ \\ ${ }^{1}$ Mathematics Department (FIQ), Universidad Nacional del Litoral, Santa Fe, Argentina \\ ${ }^{2}$ CIMEC-INTEC, CONICET, UNL, Santa Fe, Argentina (sonzogni@intec.unl.edu.ar)
}

\begin{abstract}
The technique of the Composite Mesh has been introduced in the 90's, as it can be seen in papers by the authors in the IV WCCM held in Buenos Aires. Since then some developments and applications have been made in several kinds of problems.

The technique -inspired in the mixtures theory of composite material-is based on the utilization of two finite element meshes of different element size sharing the whole domain. Each mesh is endowed with a participation factor. The composite mesh may be used for two different aims. One of them is to provide a posteriori error estimates, and the other one is to reduce the discretization errors thus leading to improved numerical solutions.

For the first case, it is shown that the residues at the connecting points of both meshes serve as a posteriori error estimates being able to detect the regions where mesh adaptation should be conducted. This aspect has been tested in different kind of problems and compared with other error estimates.

The composite mesh may be used also to provide an improved numerical solution. This is performed by a suitable choice of the participation factors. Important reduction in the discretization errors has been obtained for a wide class of problems, including those with border singularities. This improved solutions are obtained without increasing the computational cost as the problem size to be solved is of the same order of that for the fine element mesh.

Applications have been made to elliptic and parabolic problems in regular domains or in the presence of border singularities. Problems from structural mechanics (elastic analysis of plain stress and plates), as well as catalytic chemical reactors have also been addressed.

It can be mentioned that the technique of the composite mesh has been also applied in the frame of multigrid solving, showing interesting reductions in the solution errors, and making use of the fact that mesh with different size are available in the process.

In this paper we make a review of the advances in this technique and present some results on different application areas. We include some results both on error estimation and on solution improvement
\end{abstract}

Keywords: A posteriori error estimation, Composite mesh method. Improved numerical solutions 


\section{INTRODUCTION}

The finite element method is a well consolidated tool for engineering analysis. It provides an approximate solution, the accuracy being governed by parameters like the mesh size or the order of the interpolating functions. The method, to be fully useful, should be able to estimate the errors of the solution. Great effort has been made by the research community in order to develop reliable estimators and practical solution improvement techniques [12, $13,11]$. In seeking error estimates for finite elements, the use of composite meshes has been proposed [9]. It has been realized that this approach could be useful also to improve the numerical solution $[9,2]$.

The idea of the method came from the mixture theory for materials composed of several phases. In the mixture theory the properties of each component of multiphase materials enters into the formulation affected by a participation factor [6]. For example a saturated porous soil (water saturated sand soil) is composed of water and sand, and the porosity is the participation factor of water, the soil component being its complement. The physical properties (density, elastic constants, etc.) of each component enter into the formulation multiplied by the participation factor.

Based on the idea of composite materials, we introduced the use of composite finite element models where meshes with different granularity share the same problem domain. The different behaviors of each component mesh are due to the differences in the model accuracy rather than in the material properties. We can think of two finite element meshes bonded together and participating in some proportion. The results are expected to be, in a sense, intermediate between those of the analyses performed with each mesh alone. This is confirmed in the practice, and the difference in the behavior of each component mesh may be used to estimate the approximation errors.

A short description of the composite mesh concept is given in the next sections. Then application to numerical solution improvement are shown for different kind of problems. Finally applications to error estimates are briefly shown.

\section{COMPOSITE MESH}

Let $T_{h}$ and $T_{H}$ two finite element meshes with element size $h$ and $H$ respectively, with common nodes. In practice $T_{h}$ may be defined as uniform refinement of $T_{H}$, where $h=H / 2$, for instance. These meshes are overlapped over the whole problem domain with participation factors $\alpha$ and $(1-\alpha)$, the former corresponding to the fine mesh ( $h)$ and the latter to the coarse one ( $H$ ). The case $\alpha=1$ leads to the original problem with the fine mesh, whereas $\alpha=0$ to the corresponding problem with the coarse mesh [2], [8].

Let $\mathbf{A}_{h} \mathbf{u}_{h}=\mathbf{f}_{h}$ be the algebraic equation system to be solved once the finite element method is applied using the fine mesh $T_{h}$ and $\mathbf{A}_{H} \mathbf{u}_{H}=\mathbf{f}_{H}$ the corresponding system for mesh $T_{H}$. The discrete operator $\mathbf{A}_{H h}$ is defined as the matrix $\mathbf{A}_{H h}=\mathbf{P}^{T} \mathbf{A}_{H} \mathbf{P}$, being $\mathbf{P}$ a suitable prolongation operator:

$$
\mathbf{P}: \mathbb{R}^{n_{H}} \rightarrow \mathbb{R}^{n_{h}}
$$

$n_{h}>n_{H}$ being the dimensions of the discrete spaces associated to both meshes. 
A simple way of constructing $\mathbf{A}_{H h}$ is padding with zeros matrix $\mathbf{A}_{H}$ in positions associated to non common nodes, so as to get a matrix with the same dimension of that for the fine mesh. In analog way the vector $\mathbf{f}_{H h}$ may be defined.

The numerical solution for the composite mesh $\mathbf{u}_{c}$ may be obtained from

$$
\left(\alpha \mathbf{A}_{h}+(1-\alpha) \mathbf{A}_{H h}\right) \mathbf{u}_{c}=\alpha \mathbf{f}_{h}+(1-\alpha) \mathbf{f}_{H h}
$$

The asymptotic error of the finite element approximation has the form [9]

$$
\left\|u-u_{h}\right\|=C h^{p}+\mathcal{O}\left(h^{q}\right)
$$

$C$ being a constant independent of $h$, but depending of $u$ and its regularity; and being $q>p$. An extrapolation analysis of the error allows us to obtain the following estimation $[2,9,10]$.

$$
\alpha=\frac{(H / h)^{p}}{(H / h)^{p}-1}
$$

Such a participation factor allows to get improved numerical solutions for the nodal values.

Discrete norms $l_{\infty}\left(\|\mathbf{a}\|_{\infty}=\max _{i}\left|a_{i}\right|\right)$ and euclidean $\left(\|\mathbf{a}\|_{2}=\left(\sum_{i} a_{i}^{2}\right)^{1 / 2}\right)$ has been used to measure solution errors.

\section{APPLICATION OF THE COMPOSITE MESH FOR IMPROVED SOLUTION}

In this section some application are shown of the technique of the composite mesh where suitable participation factors are selected so as to minimize the discretization errors.

Following equation (4) the optimal participation factor when $H / h=2$ is $\alpha=\frac{4}{3}$. This theoretical result is confirmed with numerical experience [9, 7] and $\alpha=\frac{4}{3}$ is a good choice allowing to get precise numerical solutions. In fact this is true for regular problems. In the presence of border singularities or discontinuous parameters, the optimal value for $\alpha$ is different. However $\alpha=\frac{4}{3}$ led to improved solutions even for these non regular problems.

\subsection{Elliptic problems}

The first problem to be shown is a Poisson problem over the unit square

$$
\begin{array}{rll}
-\Delta u=f & \text { in } & \Omega \subset \mathbf{R}^{2} \\
u=0 & \text { in } & \Gamma(=\partial \Omega)
\end{array}
$$

where $\Omega=(0,1) \times(0,1)$ and $f(x, y)$ is a polynomial function.

The analytical solution is shown in (Fig. 1).

The point wise exact error for a standard finite element mesh with $40 \times 40$ elements is shown in figure 2 . Figure 3 plots the point wise errors for a composite mesh where the fine 


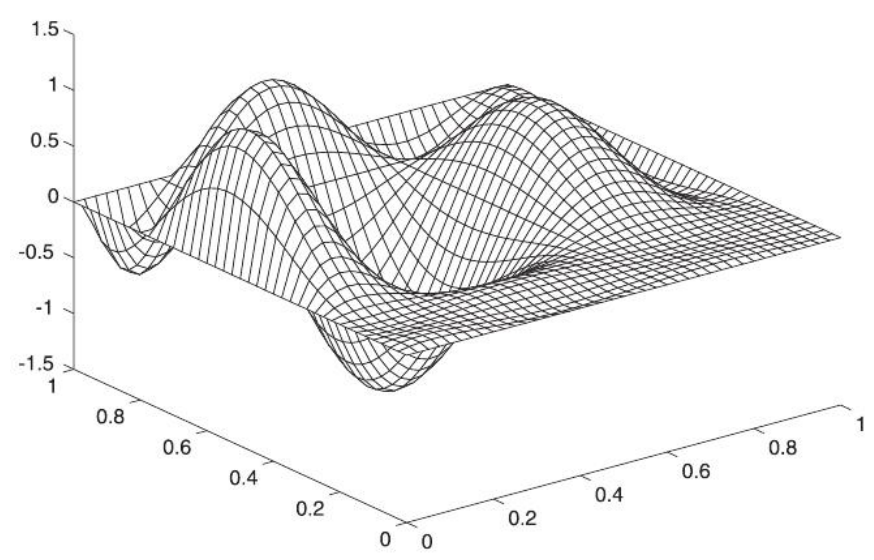

Figure 1. Analytical solution $u(x, y)$ of problem (5)

mesh has $40 \times 40$ elements while the coarse one $20 \times 20$ elements. Figure 4 shows the same errors but the plot has been restricted to common nodes.

The discrete global errors, measured in $l_{2}$-norm and $l_{\infty}$-norm are shown in figure 5 versus the number of elements (the number of elements of the coarse mesh, in the case of the composite mesh).

The order of the convergence for the composite mesh is nearly twice that for the standard element meshes.

The example presented is representative of other elliptic problems. In particular 2D and $3 \mathrm{D}$ problems have been tested, as well as diffusive and advection-diffusion problems with boundary layers [7].

\subsection{Problems with singularities}

The order of problems with singularities deteriorates and the estimation made with equation (4) is not straightforward.

For a square problem domain with a variable angle $\beta$ (Fig. 6) the optimum value for $\alpha$ ranges from $\alpha=\frac{4}{3}$ to roughly $\alpha=1.7$ (Fig. 7 [7]). However, even in the worst case, a value

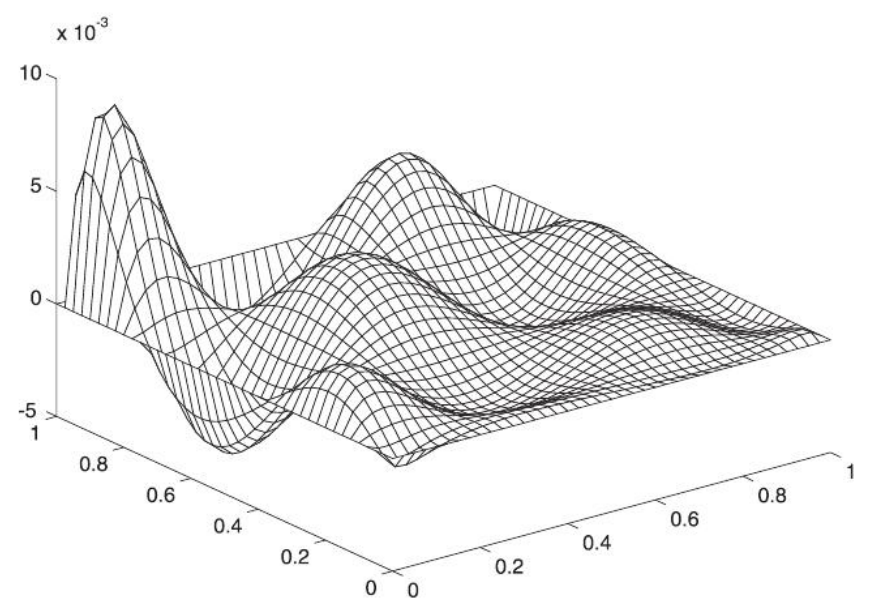

Figure 2. Exact error of the standard finite element solution with $40 \times 40$ elements. 


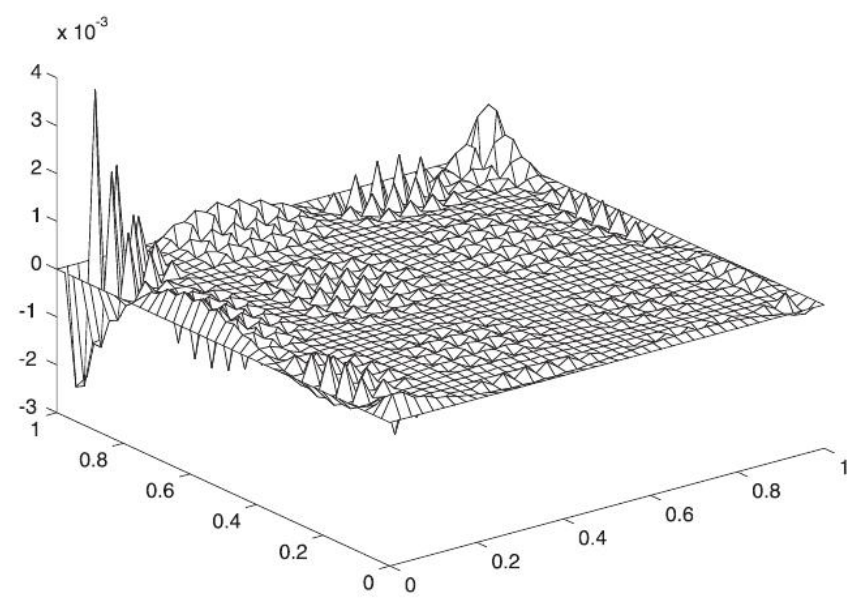

Figure 3. Exact error of the composite mesh solution combining $40 \times 40$ and $20 \times 20$ elements

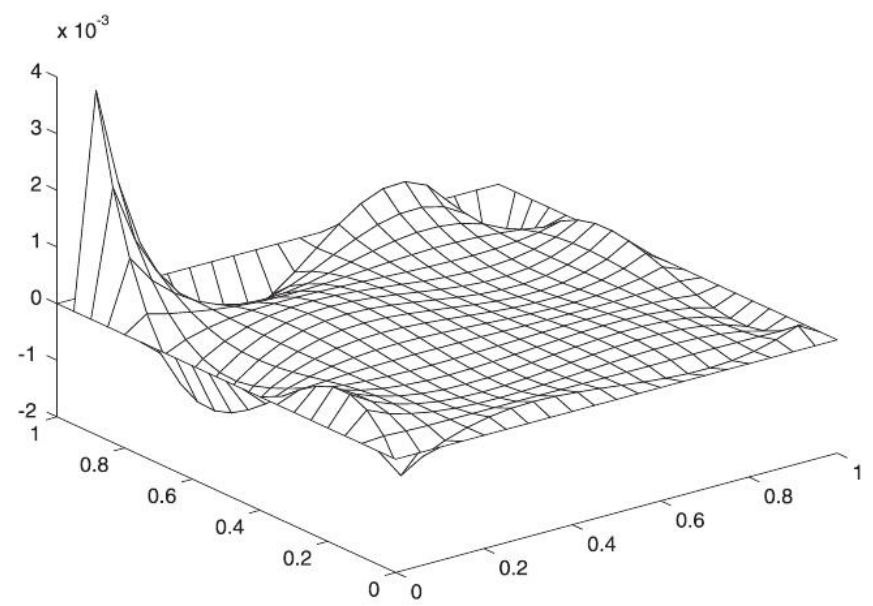

Figure 4. Exact error of the composite mesh solution: combining $40 \times 40$ and $20 \times 20$ elements. Function restricted to common nodes.

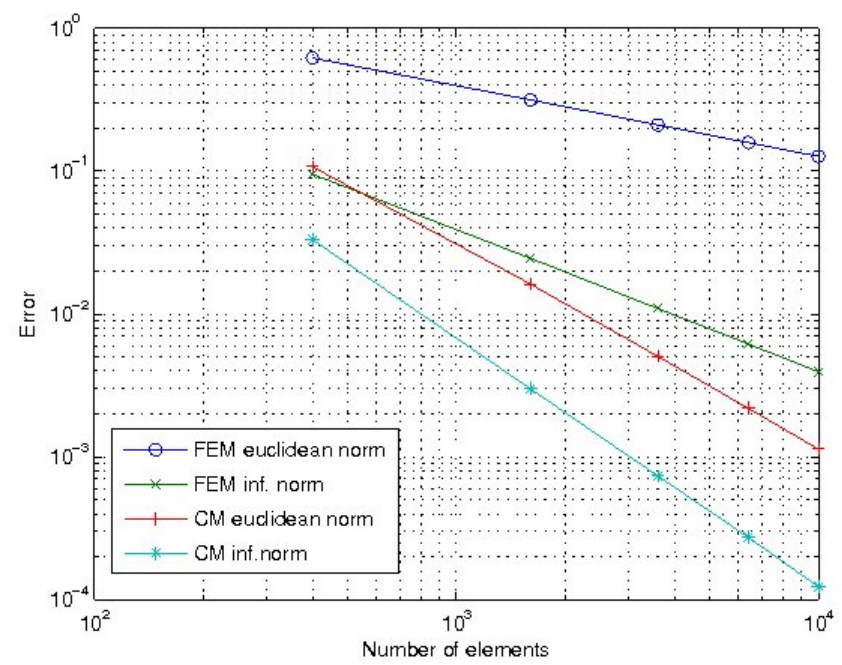

Figure 5. Discrete norm errors for Poisson problem. (5) 


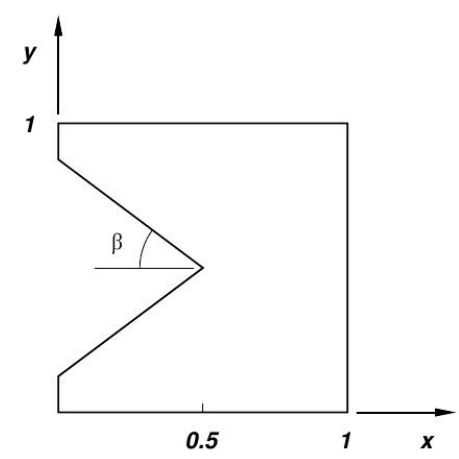

Figure 6. Problem with border singularity

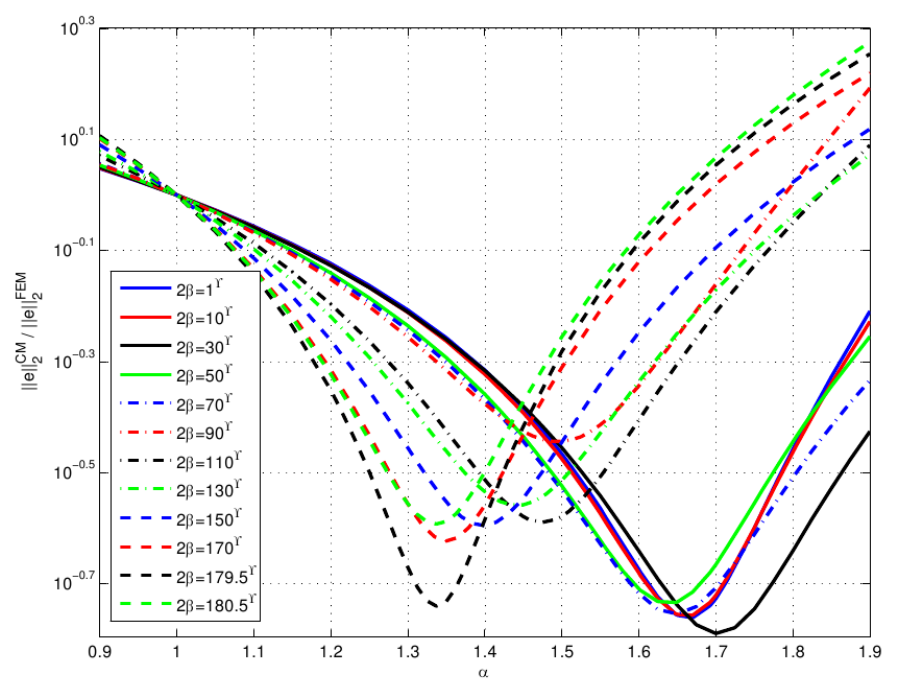

Figure 7. Optimum $\alpha$ as a function of singularity angle $\beta$ in problem 6 (taken from [7]).

$\alpha=\frac{4}{3}$ leads to a better solution than that of the standard finite element case, as can be seen in the same figure.

\subsection{Problems in linear elasticity}

A number of problems of the static response of linear elastic structures have been solved.

As a first problem a plane stress cantilever beam is shown (Figure 8). This problem has been chosen because, for the particular boundary conditions, the exact solution is known and it has been used in the literature for error estimation. The square beam of linear elastic material is loaded with shear forces.

Here two kinds of solutions have been obtained: a standard finite element solutions and finite element composite mesh solutions.

The beam was modeled with linear rectangular finite elements. Results have been obtained for meshes with increasing number of elements, from $2 \times 2$ elements, up to $64 \times 64$ elements. Fig 8 shows one of such meshes.

The analyses for the composite mesh have been performed with participation factors $\alpha=\frac{1}{2}$ and $\alpha=\frac{4}{3}$. The case $\alpha=\frac{1}{2}$ was expected to give worse results for the same problem 

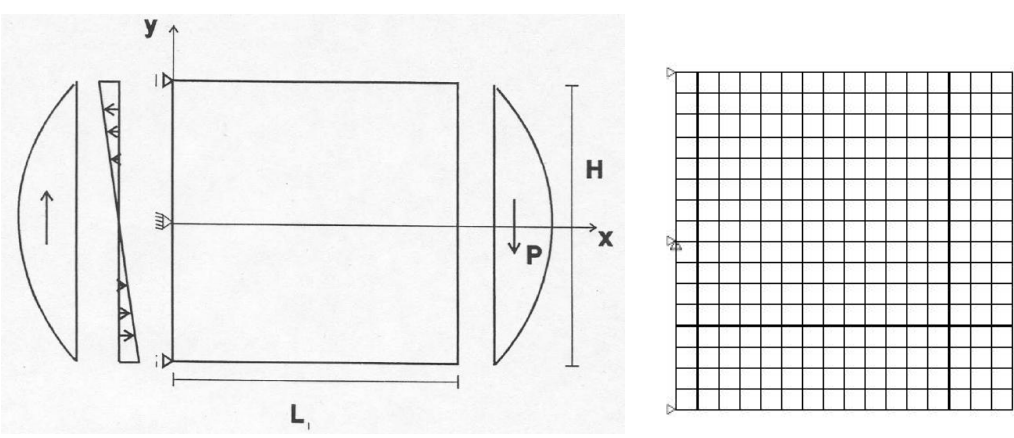

Figure 8. Plane stress cantilever beam. Scheme of the problem and one of the FE meshes.

size since the coarse mesh is added to the fine mesh in the same proportion. The case $\alpha=\frac{4}{3}$ was expected to improve the results as in the case of the previous example.

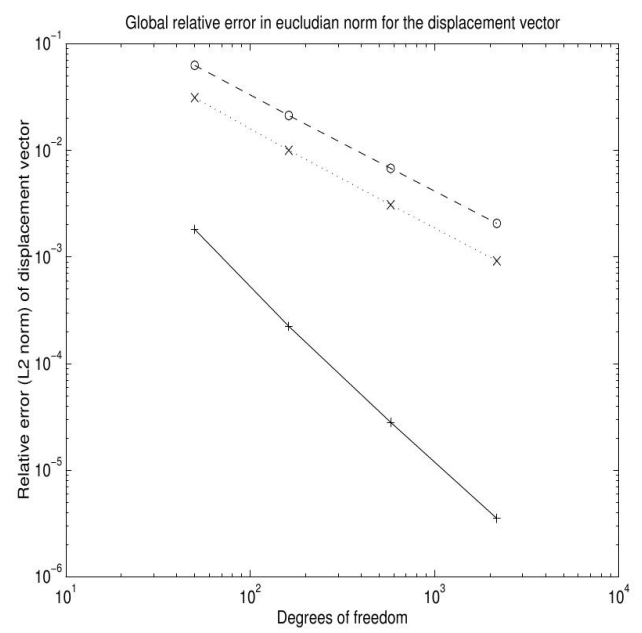

Figure 9. Cantilever beam. Global relative error in the euclidean norm for the displacement vector. (x) Simple mesh; (o) Composite mesh $\alpha=0.5$; (+) Composite mesh $\alpha=\frac{4}{3}$

The results with different mesh size are summarized in the following figures, for three cases: standard finite element mesh; composite mesh with $\alpha=\frac{1}{2}$; and composite mesh with $\alpha=\frac{4}{3}$. Global relative errors measured, in the euclidean norm and in the infinity norm, of the displacement vector are shown in Fig 9 and Fig 10, respectively.

As a second problem of linear elastic analyses the case of a plane stress distribution on a cracked domain is shown. A strong singularity is present in this case. The problem is defined on a square domain, which by symmetry, represents a half cracked membrane (Fig 11).

The reference in this case was a standard finite element solution with a fine mesh comporting 16178 degrees of freedom, refined near the singularity point. Figure 12 shows results in global relative error, measured in infinity norm, of the displacement vector obtained with: standard finite element; composite mesh with $\alpha=0.5$; and composite mesh $\alpha=\frac{4}{3}$. In spite of the singularity, the composite mesh with $\alpha=\frac{4}{3}$ greatly reduces the global error.

As a third problem results for a thin plate are shown. In this case, solved by [1], thin plates modeled by Kirchoff theory, are studied with the composite mesh technique. Again $\alpha=\frac{4}{3}$ has been used as participation factor. Figure 13 shows the results for a simply supported square plate with uniform transversal load. The central displacement is compared to that for 


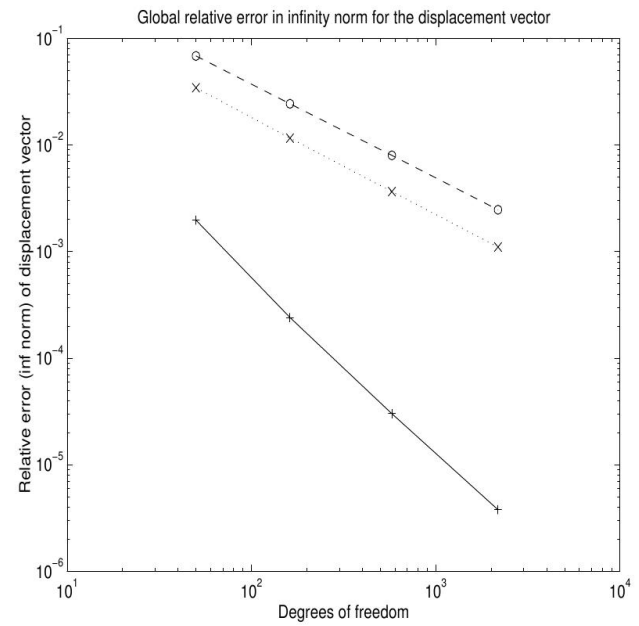

Figure 10. Cantilever beam. Global relative error in infinity norm for the displacement vector. (x) Simple mesh; (o) Composite mesh $\alpha=0.5$; (+) Composite mesh $\alpha=\frac{4}{3}$
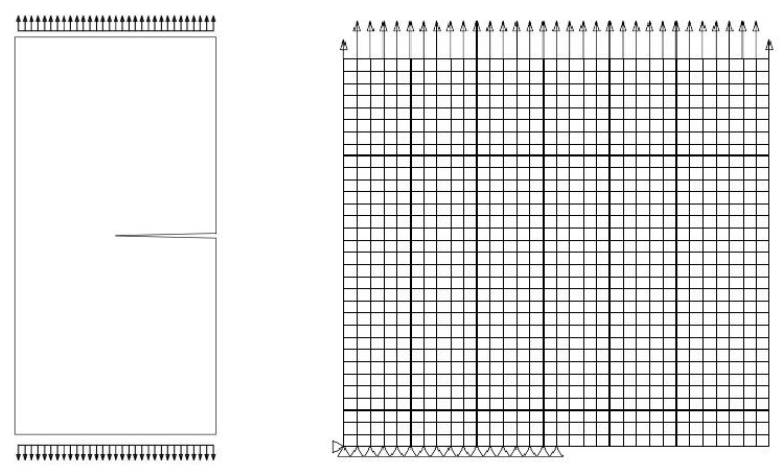

Figure 11. Plane stress cracked membrane and one of the FE meshes for the half domain.

a $128 \times 128$ element mesh. Diamonds stands for simple finite element mesh and triangles for the composite mesh. As in the previous examples, a better result has been obtained with the composite mesh for the same problem size.

The composite mesh technique has also been applied to eigenvalue problems. A simple problem of free vibration of a bar in its axial mode has been studied and the results are summarized in figure 14. The main vibration frequency is plotted vs the mesh size. Two curves are shown, one for a standard finite element and the other for a composite mesh with a participation factor $\alpha=\frac{4}{3}$. It can be seen here again the improvement for the composite mesh results.

\section{APPLICATION OF THE COMPOSITE MESH FOR ERROR ESTIMATION}

Using the composite mesh with $\alpha=0.5$ the result is worse than that of a simple mesh $(\alpha=1)$. However the residues at common nodes may serve as error estimators.

We define the residues as

$$
\mathbf{r}_{H}=\mathbf{A}_{H} \mathbf{u}_{c}^{*}-\mathbf{f}_{H}
$$




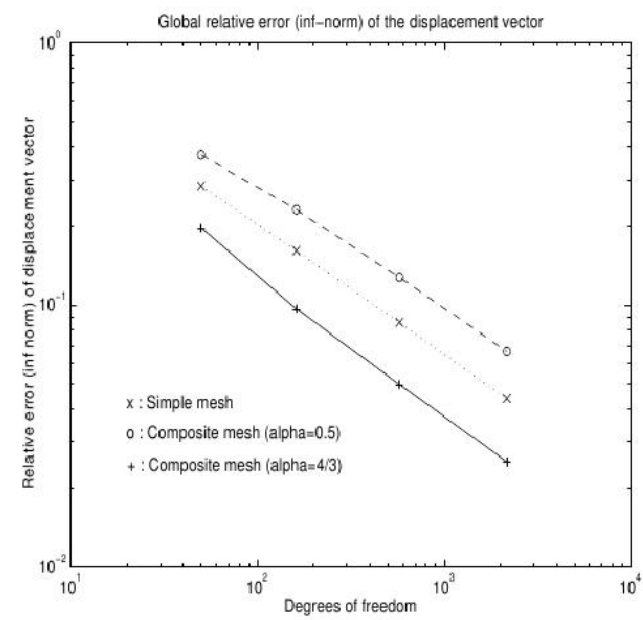

Figure 12. Global relative error of the displacement vector in infinity norm. (x) Simple FE mesh; (o) Composite mesh $\alpha=0.5$; (+) Composite mesh $\alpha=\frac{4}{3}$

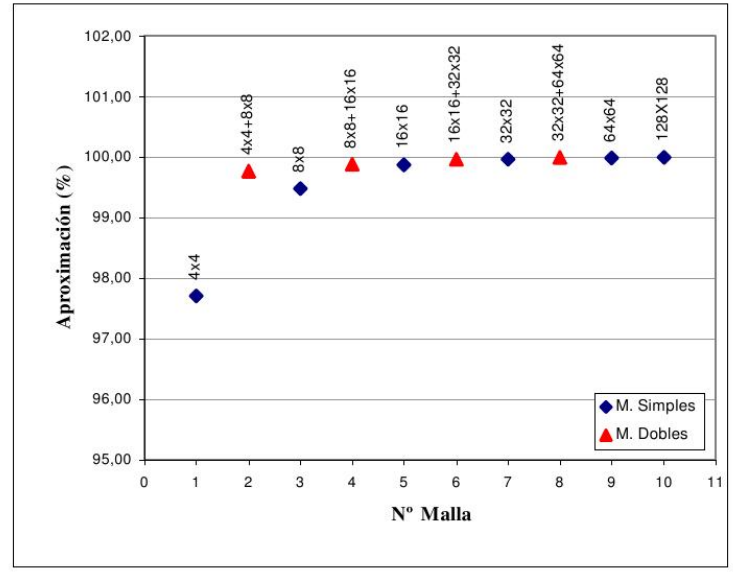

Figure 13. Results for a square simply supported plate. Relative errors in infinity norm for different mesh granularity. $\diamond:$ simple mesh; $\triangle$ : composite mesh.

being $\mathbf{u}_{c}^{*}$ the solution of eq (2) restricted to nodes of the coarse mesh.

It has been observed that residues may give an approximate distribution of the discretization errors. In this case there have been used to detect zones where refine the mesh in adaptive process.

Figure 15 shows the map of the exact errors and residues for a Poisson problem on a square domain [5]. As can be seen there is a similar pattern between both maps.

In the same paper a problem with border singularity is treated. In this case a problem on a L-shaped domain is studied. Figure 16 plots the order of the method in the neighborhood of the singularity point. A plot of the exact errors an residues is given in figure 17.

A problem of a monolith catalytic reactor has been studied in [4]. It is modeled on a square domain by systems of partial differential equations representing the mass and energy balances inside the reactor. One of the unknowns is the concentration of a chemical species inside the reactor. The error in the finite element solution for concentration is shown in figure 18 computed via residues of the composite mesh, and computed with the Zienkiewicz-Zhu estimator [12]. 


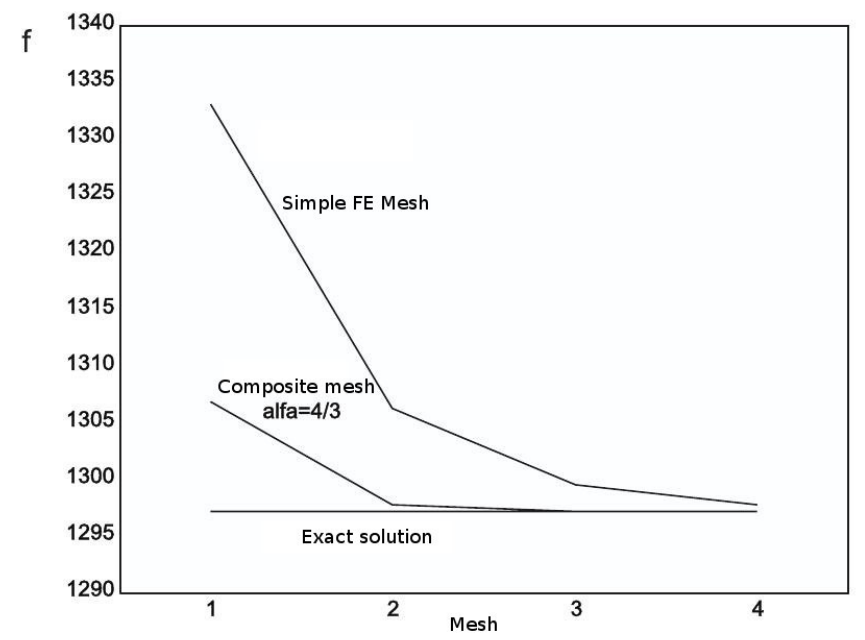

Figure 14. Frequency of the first mode in axial vibration of an elastic bar.
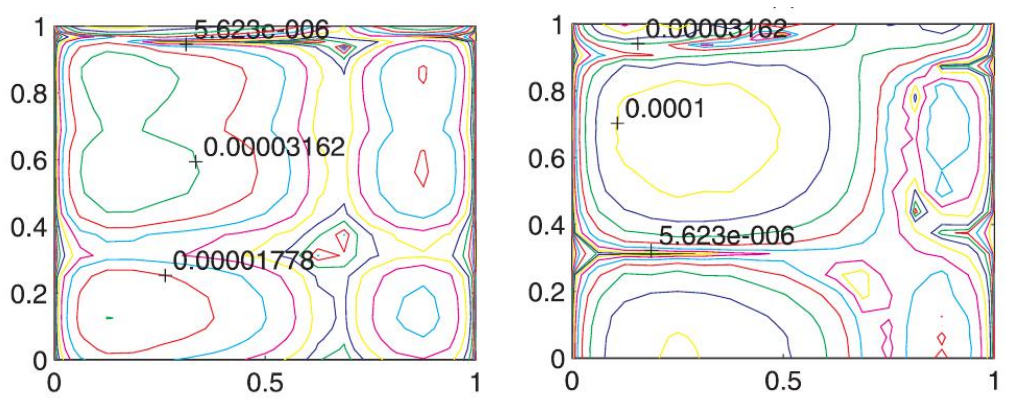

Figure 15. Exact errors (left) and residues (right) for a Poisson problem on a square domain

For the problem of cracked membrane (Figure 11) which has also a severe singularity, the residues of the composite mesh has been computed. They are plotted in figure 19 together with a plot of the principal stresses. As expected the residues, marking the discretization errors, are placed in the neighborhood of the singularity.

Finally the application of the composite mesh to a parabolic problem is shown. This case treated in reference [3] and stands over a square domain. A map of the exact errors and the residues is given in figure 20. It can be seen again a reasonable agreement between both plots.

\section{CONCLUSIONS}

Some applications of the technique of composite finite element are shown in this papers. This technique easy to apply is based on placing two finite element meshes sharing the problem domain. These component meshes have different intrinsic accuracies and are affected each by a weight or participation factor. The composite mesh has been used for two purposes: to estimate a posteriori discretization errors or to improve our finite element solution.

In the first case, with a simple matrix vector operation involving the vector of nodal values, one can get a map o residues which gives an idea of the distribution of the discretization errors and may serve to conduct adaptive refinement.

In the second case, adopting a suitable participation factor an improved solution may 


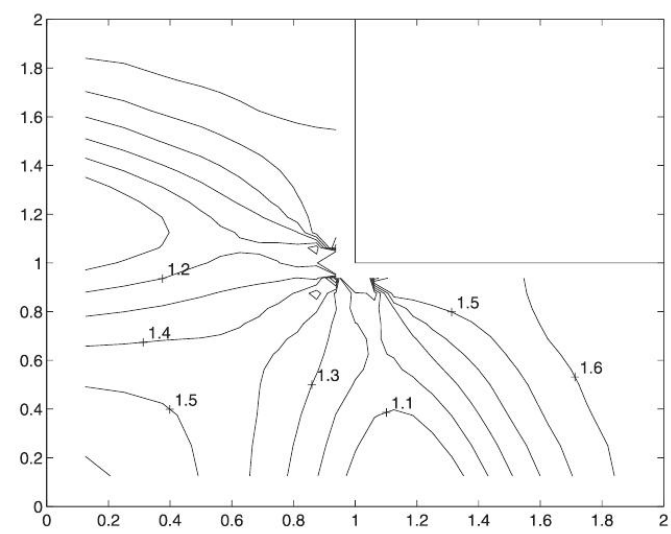

Figure 16. Poisson problem on a L shaped domain. Orders of the method
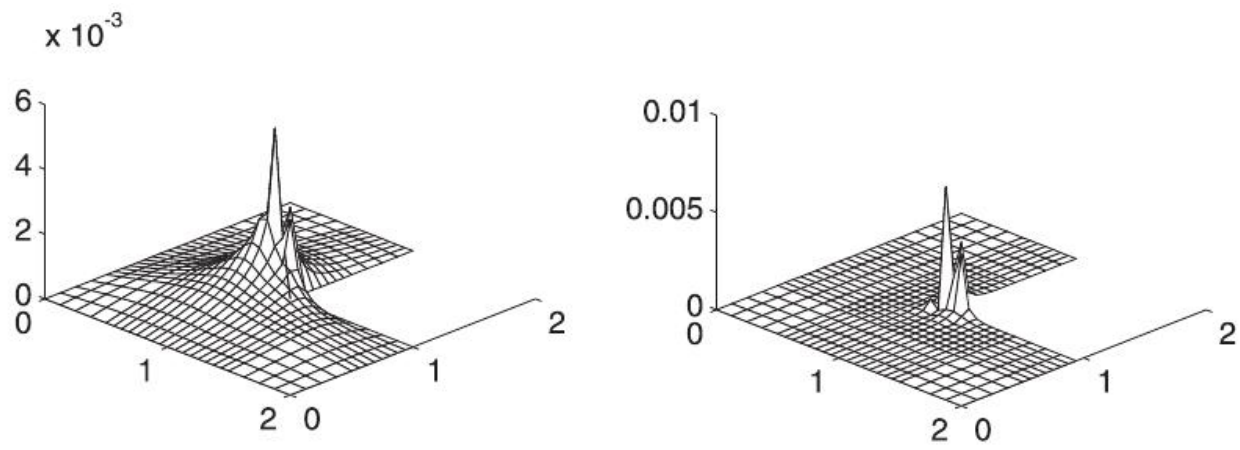

Figure 17. Poisson problem on a L shaped domain. Exact errors (left) and residues (right)

be obtained. Important reduction in the discretization errors may be obtained without any post-processing of the results.

\section{Acknowledgements}

This papers received support from the National Agency for Promotion of Science and Technology (ANPCyT) through grants PICT 2006/1506 and PICT-2010/2492, from CONICET through grant PIP 112-200801-2956, and from National University of Litoral through grant CAI+D 2009-III-4-2.

\section{References}

[1] H. F. Begliardo and V. E. Sonzogni. Aplicaciones del método de doble malla a problemas de elasticidad bidimensional y placas delgadas. In Proc. MECOM 2002: VII Congreso Argentino de Mecánica Computacional, Santa Fe, Argentina, 28-31 October, pages 1370-1381, 2002.

[2] M. Bergallo, C. Neuman, and V. Sonzogni. Composite mesh concept based fem error estimation and solution improvement. Computer Methods in Applied Mechanics in Engineering, 188:755-774, 2000. 

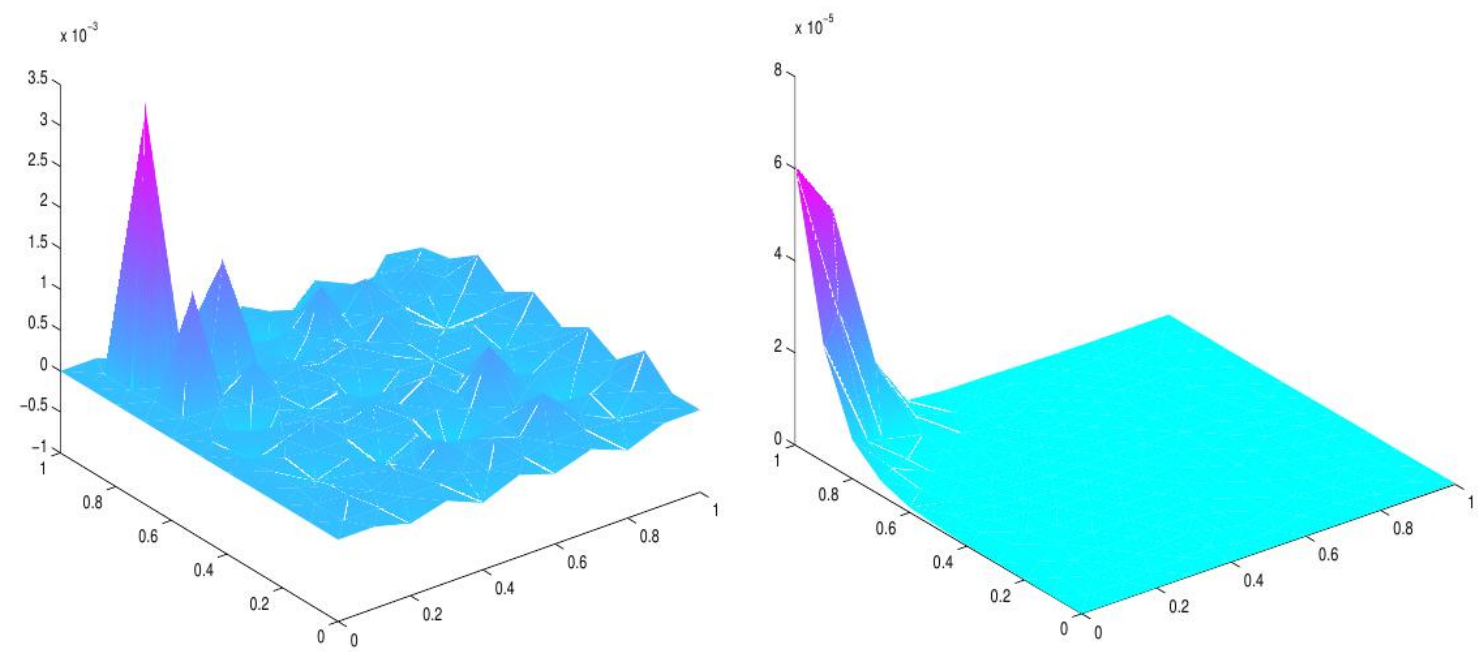

Figure 18. Reactor problem. Residues (left) and Zienkiewicz-Zhu estimators (right)
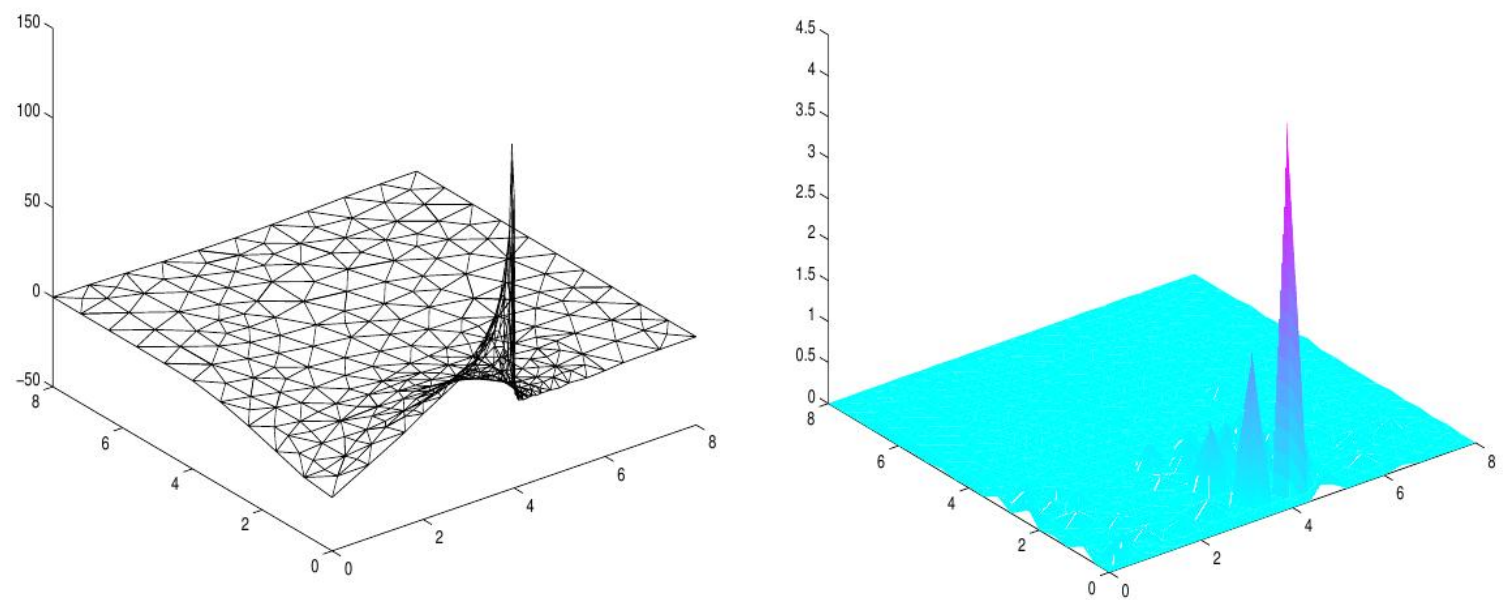

Figure 19. Problem of cracked strip. Principal stresses (left) and residues (right)

[3] M. Bergallo, C. Neuman, and V. Sonzogni. Composite mesh fem for parabolic evolution problems. In Proc. ECCOMAS 2000: European Congress on Computational Methods in Applied Sciences and Engineering, Barcelona, 11 al 14 de setiembre, 2000.

[4] M. B. Bergallo, C. E. Neuman, and V. Sonzogni. Elliptic problems with essential border singularities: Improved solution and qualitative error estimation. In Proc. ENIEF'94: Congreso sobre Metodos Numericos y sus Aplicaciones, Bariloche, 8-11 noviembre, pages 2979-2998, 2004.

[5] M. B. Bergallo, C. E. Neuman, and V. E. Sonzogni. A finite element error estimation based on the mixed mesh concept. In Proc. IV World Congress in Computational Mechanics, Buenos Aires, Argentina, June 29-July 2, 1998.

[6] J. H. Prevost. Mechanics of continuous porous media. International Journal in Engineering Science, 18:787-800, 1980.

[7] S. S. Sarraf. Análisis y generalización de la técnica de la malla compuesta y su in- 

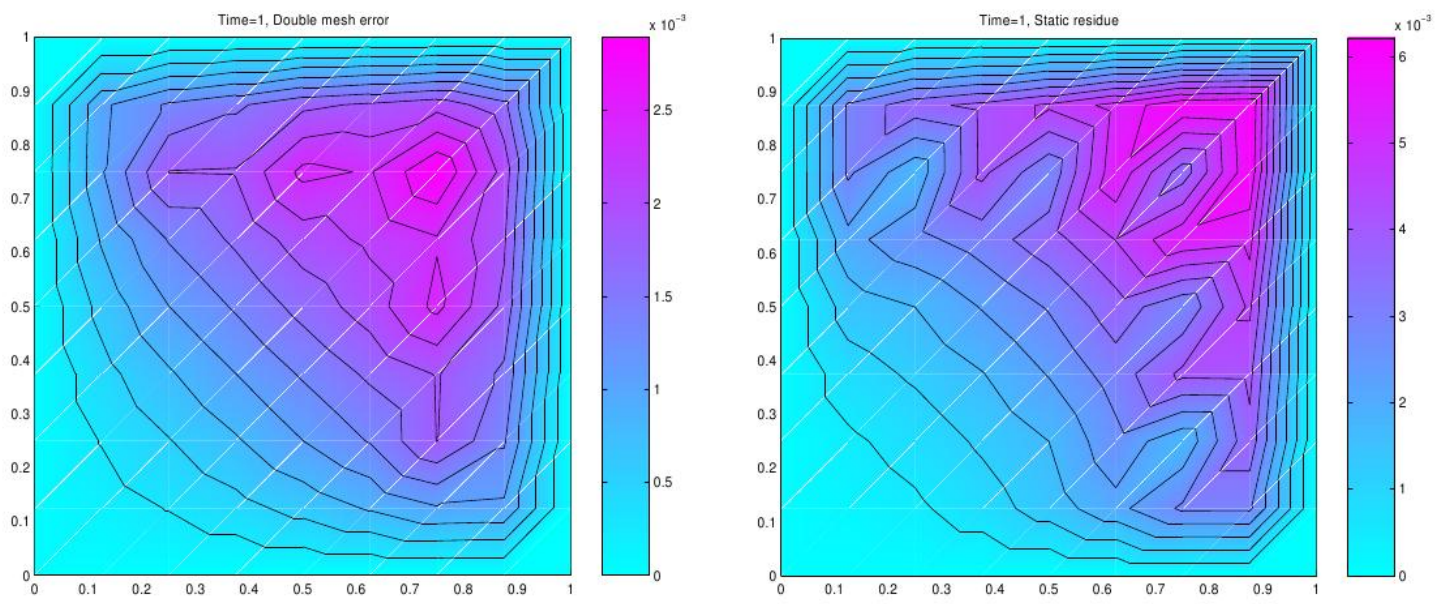

Figure 20. Parabolic problem. Exact errors (left) and residues (right) for a given time

tegración con el método multigrilla. Tesis doctoral, Universidad Nacional del Litoral, 2011.

[8] S. S. Sarraf, M. B. Bergallo, and V. Sonzogni. Integración de la técnica de malla compuesta con el método multigrilla. In Proc. MECOM 2010, 2010.

[9] V. E. Sonzogni, M. B. Bergallo, and C. E. Neuman. Uso de una malla compuesta para estimar errores de discretización y mejorar la solución en elementos finitos. In Proc. MECOM 1996, pages 123-132, 1996.

[10] S. Toro, V. Sonzogni, and C. Neuman. Elementos finitos de diferentes ordenes para problemas de elasticidad plana y mezclas de sus mallas. In Proc. MECOM 2005: VII Congreso Argentino de Mecánica Computacional, Buenos Aires, 16-18 noviembre, pages 3171-3186, 2005.

[11] R. Verfhurt. A Review of A Posteriori Error Estimation and Adaptive Mesh-Refinement Techniques. Wiley -Teubner, Chichester, 1996.

[12] O. C. Zienkiewicz and J. Z. Zhu. A simple error estimator and adaptive procedure for practical engineering analysis. International Journal on Numerical Methods in Engineering, 24:337-357, 1987.

[13] O. C. Zienkiewicz and J. Z. Zhu. Adaptativity and mesh generation. International Journal on Numerical Methods in Engineering, 32:783-810, 1991. 